\title{
Internet Districts in Italy: Is Proximity an Antidote against Weak Broadband Competition?
}

\author{
Alessio D'Ignazio ${ }^{1}$, Emanuele Giovannetti $^{{ }^{*}}$ \\ ${ }^{1}$ Trinity Hall College, Cambridge,United Kingdom \\ ${ }^{2}$ LAIBS, Anglia Ruskin University, Cambridge, United Kingdom, \\ and Department of Economics, University of Verona, Italy \\ E-mail: emanuele.giovannetti@anglia.ac.uk \\ Received January 18, 2011; revised April 2, 2011; accepted April 15, 2011
}

\begin{abstract}
We study the effects of proximity in the interconnections between Internet providers participating at the three main Italian Internet Exchange Points. We find that geographical proximity plays a role in driving Internet Providers' interconnection, positively affecting the likelihood of an agreement. This suggests the existence of localized positive externalities in the form of mutual knowledge and reputation, one of the cited drivers underlying the formation of districts in non high-tech industries. We interpret these findings as resulting from the necessity for cooperation in interconnection between smaller providers facing the weak competitiveness of the highly concentrated Italian broadband access market.
\end{abstract}

Keywords: Internet, Peering, Clustering, Interconnection, Competition in Networks

\section{Introduction}

The policy recognition of the role played by Information and Communication Technologies (ICT) in driving growth is, by now, well established. In 2005, the European Commission launched 2010 , a new initiative to accomplish the target of a fast growth for the economy and to improve the European competitive position, following the Lisbon National Reform Programs (March 2000). The 2010 initiative identifies key actions within the ICT sector: the driving factor is the convergence of communication services (data, voice and video), earlier available only through distinct ICT devices. The effects of the convergence are tangible, with an increase of the sales of electronic devices, the exceptional development of the online content markets, as for example with the launch of the Internet Protocol Television (IPTV), and the fall in the telephone calls prices ${ }^{1}$ due to the use of the Voice over Internet Protocol Technology (VoIP). This convergence is also generating increased demand for broadband: in the period between January 2006 and January 2007 a total of $21,058,525$ new broadband lines were added, an increase of $35.70 \%$ in the EU countries. ${ }^{2}$ This growth impetus is however showing the first signs of

\footnotetext{
${ }^{1}$ Despite the general decline in prices, tariffs still vary widely among countries.

${ }^{2}$ See $[1]$.
}

slowing down: the increase in new broadband lines between January 2009 and January 2010 has been of a total of $10,292,727$, an increase of $8.31 \%$ in the EU countries. Worryingly enough, this slow down in broadband lines growth rate is taking place at an average penetration rate still quite low, at $24.8 \%$ in the EU countries.

The EU broadband growth process has been largely unbalanced, with some of the well-performing countries growing faster, and others lagging behind, this was particularly the case for Italy. While starting in January 2009 with broadband penetration rates $(19.8 \%)$ lower than other similar countries, and than the EU average, see Figure 1 below, Italy also achieved a lower than average growth in penetration rates in $2010^{3}$. Asymmetric broadband penetration and growth, as shown by these data, are likely to be the outcome of underlying national features of the modalities, speed, pricing and infrastructures related to the Internet access.

In May 2010 the European Commission adopted, within the Europe 2020 strategy, the Digital Agenda, aiming at boosting the digital economy and strengthening competition in the ICT sector.

\footnotetext{
${ }^{3}$ In January 2010 the broadband penetration rate reached $20.64 \%$ in Italy, about 10 percentage points below Germany, France and UK. This slower process dates back many years; for example in 2008 the penetration rate in Italy was about $17.1 \%$, against $23.8 \%$ in Germany, $24.8 \%$ in France and $25.7 \%$ in the UK.
} 


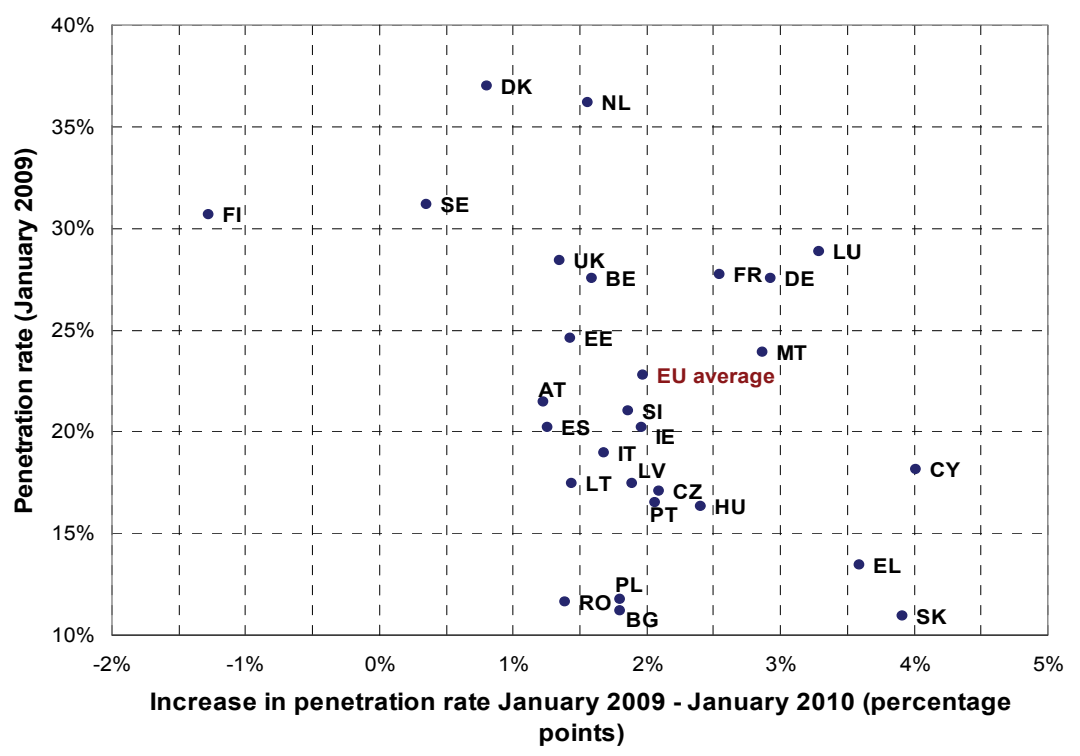

Figure 1. EU Penetration rate and speed of progress.

The success of the Digital Agenda relies on the possibility of achieving a greater quality of the services offered, with a parallel reduction in the connectivity costs. A key role in this process is then played by our object of study, the Internet Exchange Points (IXPs), physical networks infrastructures that facilitate the traffic exchange between the Internet Service Providers ${ }^{4}$.

In this paper, we focus in particular on the interconnection decisions between operators participating at main Internet Exchange Points (IXP) in Italy: the Milan Internet Exchange Point (Mix), the Turin Piedmont Internet Exchange Point (Topix) and the Nautilus Mediterranean Exchange Point (NaMex) in Rome. Almost the entire Italian Internet traffic is routed through these exchanges, where also a relevant part of the country international connectivity takes places. Moreover, these IXPs have also played a crucial role both in developing the backbone of the Italian e-Government system and as country's innovation incubators.

Our focus is to assess the role played by geographical proximity in shaping the incentives of the Internet Service Providers (ISPs) interconnection strategies in Italy.

We assess the specific role of geographical distance on interconnection by estimating a binary model of the different factors affecting the probability that two ISPs will set up a direct interconnection.

Our results show that the distance between two ISPs consistently affects their interconnection decisions, by reducing the likelihood of them reaching a direct agreement. We interpret this negative effect, exerted by distance on interconnection, as evidence of localized positive external-

\footnotetext{
${ }^{4}$ An Internet exchange point (IXP) is a physical infrastructure that allows different Internet Service Providers (ISPs) to exchange Internet traffic by means of direct interconnection agreements.
}

ities, due to mutual knowledge and reputation effects, one of the most cited drivers underlying the formation of industrial districts also in non high-tech industries.

The negative role played by distance in establishing direct interconnections points towards an emergent district-like morphology for the Internet upstream sector, the underlying infrastructure for High-tech economic activity. Moreover we read this emerging morphology as the result of a collective interconnection strategy followed by smaller ISPs to counterbalance the market power asymmetry existing between incumbent and smaller entrants, expressed by the high concentration of the Italian broadband access market. It is indeed hardly surprising that, with a former monopolist detaining almost $60 \%$ of the market share, smaller competitors will benefit from reciprocal proximity as a coordinating device in facilitating the emergence of cooperative institutions for the reciprocal routing of the Italian Internet traffic, and by so doing facilitating the bypass of existing infrastructural bottlenecks.

The rest of the paper is organized as follows. Section 2 discusses Internet trends in Italy, while Section 3 introduces the morphology of interconnection agreements within Italian IXPs and analyzes the determinants of these bilateral relationships. Finally, Section 4 concludes.

\section{Internet in Italy}

\subsection{Internet Penetration}

The geographical heterogeneity in the European Internet penetration is also reflected in Italy, where broadband penetration is still relatively low if compared with other European countries, such as UK and France. 
The broadband offer in Italy is itself highly unbalanced: out of the 12,395,106 broadband estimated connections in 2010 , only $390,617(3.2 \%)$ were based on optical fiber, while $12,004,489$ were based on xDSL technology, i.e. still relying on the pre-existing telephonic infrastructure. In France, Germany and UK the share of optical fiber lines were $5.1 \%, 10.2 \%$ and $21.0 \%$ respectively.

These figures [1] can partly explain the low broadband penetration in Italy in the first years of 2000s, and are mainly due to the effective absence in Italy of a competitive cable alternative to DSL. The increased broadband penetration in Italy, though unbalanced, was facilitated by the last decade of regulatory efforts at European Level, leading to an improved implementation of the Local Loop Unbundling (LLU). Italy however still lags behind other European countries, and new investments in infrastructure are necessary to sustain broadband penetration. In particular, these investments could exploit the high mobile penetration rates, and could facilitate the diffusion of the recently introduced wireless broadband access modalities such as High Speed Downlink Packet Access (HSDPA). As it is always the case, however, any infrastructural innovation poses new interconnection issues and the need from the relevant antitrust authorities to define a clear set of rules granting a fair interconnectivity environment. This has been essential for the issues posed by the LLU, for those related to the fix/mobile interconnection agreements and it will become even more relevant for the questions raised with the diffusion of next generation networks. In this framework the ability of discriminating Internet traffic in terms of its nature, and the proposals for generating differentiated levels of services, poses even more difficult settlements and quality of interconnection issues, as discussed in the increasingly popular debate on net neutrality ${ }^{5}$. This debate also has specific policy repercussions on the break-up of the previous national telecom monopolist that often still maintains a stronghold on the main national access networks ${ }^{6}$. With regards to this, it is a clear indicator of the poor state of the Italian market competitiveness the fact that, while by 2007 in the group of $27 \mathrm{EU}$ countries the incumbent's share was $47.2 \%$ of the broadband market, and specifically the British Telecom (BT) only had $25.2 \%$ share of the UK broadband market, in Germany the incumbent share was $48.0 \%$ and in France 46.2\%; on the other hand, the share detained in Italy by Telecom Italia amounted to $66.6 \%{ }^{7}$. In 2010 this was still high, at

\footnotetext{
${ }^{5}$ The principle of Net Neutrality states that Internet users should be in control of what content they view and what applications they use on the Internet. Net neutrality is a major issue in the U.S. new telecommunications laws (see for example [2]).

${ }^{6}$ See for example the position expressed by EU Commissioner Reding on the possible necessity of structural break-up of existing dominant players [3].

${ }^{7}$ Source: [4]
}

$56.8 \%^{8}$, against $46.2 \%$ in Germany, $45.5 \%$ in France and $28.3 \%$ in the $\mathrm{UK}^{9}$.

A first sign of the need of stronger coordination among small providers in Italy was the formation of the Italian Association of Internet Service Providers (AIIP) in June 1995; AIIP plays a crucial role in coordinating its 44 member on Regulatory Issues, mainly concerning interconnection with the dominant network of Telecom Italia. A typical example of the AIIP'a pro-competitive role can be found in the antitrust case raised by this association against Telecom Italia, concluded with a sanction of 115 millions of euros for abuse of dominant position, against this operator ${ }^{10}$. Together with supporting neutral and fair interconnection to the main backbone for its members, the AIIP also promotes the multiplication of settlement free bilateral exchanges of traffic among ISPs (peering), and this usually takes place at Internet Exchange Points (IXPs).

\subsection{The Role of IXPs}

As we mentioned in the introduction a key role in achieving the objectives of the Digital Agenda can be played by the IXPs.

The first IXP to emerge in Italy was the Milan Internet Exchange ${ }^{11}$ created in 1996 by a group of ISPs operating in Italy, along the same guide lines the Roman IXP, Na-

\footnotetext{
${ }^{8}$ See [1].

${ }^{9}$ Moreover, notwithstanding this more competitive framework, to avoid the risk of a break-up possibly imposed by the UK Regulator Office of Communication (OFCOM), BT committed itself to provide market interconnection equivalence to its competitors. This was implemented by an operational separation between BT Retail and BT wholesale that, managed by an independent board of directors, will provide equal technical and commercial interconnection conditions to both BT Retail and to its competitors [5].

${ }^{10}$ In Case A351 that was decided in 2004, the ICA imposed its highest-ever fine of EUR 152 million on Telecom Italia for abuse of dominant position (approximately $5 \%$ of total worldwide turnover). In this case, Telecom Italia was considered liable for applying contractual conditions dealing with exclusive and English clauses, and of discriminatory practices against its competitors on the intermediate services markets. Telecom Italia appealed against the antitrust decision, first before the TAR Lazio, and then before the Consiglio di Stato, finally obtaining a reduction of the fine to EUR 115 million [6].

${ }^{11}$ Mix, started in 1996 as a voluntary non profit network access point, has been registered in January 2000 as a Limited company by 28 charter members, continuing however to operate as a non profit consortium. Mix is the most successful IX in Italy, with about 60 members, both Italian and international ISPs, with an aggregate traffic of up to 10 Gbps. The institutional service Mix provides is a high quality interconnection points for ISPs and Telco Operators, making them available a very reliable peering point. Having realised the essential European dimension of the Internet connectivity, the Mix became one of the funding members of the Euro-IX started in 2001 with 7 members (IXPs from Austria Belgium, Germany, U.K., Italy, the Netherlands and Sweden) having in 2007, 33 members from different European countries. Euro-IX aim is to further develop, strengthen and improve the IXP community throughout Europe. This is done by combining their resources so as to co-ordinate technical standards across the continent, develop common procedures, and share and publish statistics and other information [7].
} 
$\operatorname{mex}^{12}$ was later constituted. Later on, other Regional community networks which have also all the characteristics of an IXP emerged: Topix in Piedmont ${ }^{13}$ and Tix in Tuscany.

\section{Proximity in the Internet}

\subsection{The Internet Peering and Transit}

The Internet is composed of many independent networks of very different sizes, located around the globe, all directly or indirectly interconnected with each other. This last feature guarantees the Internet's most important property: universal exchange of traffic between all end users (universal connectivity). The industry is still mainly unregulated also due to its transnational nature, and networks are left free to decide where, how and with whom to interconnect.

Internet Service Providers (ISPs) rely on interconnections both among themselves and to larger networks for the delivery of their customers' data packets to their destinations outside the range of the ISP's own subscribers.

Two simple types of interconnection agreements have emerged to regulate traffic at exchange points between networks: transit agreements and peering agreements. In a transit agreement, a large network the transit provider - offers access to the entire Internet to a smaller customer network against the payment of a fee often related to the capacity of the connection link.

Under a peering agreement two networks exchange the traffic directed to each other's end users only. Peering can be seen as a reciprocal, non-monetary exchange relationship that often implies various forms of cooperation. Peering, when taking place privately, implies establishing direct exchange points between the two networks, with the costs of creating and maintaining the exchange points typically shared evenly. To

\footnotetext{
${ }^{12} \mathrm{NaMeX}$ was founded in 2001 in Rome as a Not-for-profit Consortium an evolution of NAP Nautilus, a spontaneous Neutral Access Point enabling 4 ISPs to exchange routes between them. NaMeX had 19 members in 2007; being the second largest IXP in Italy, with a peak aggregate traffic of 2 Gbps. NaMeX provides an ever-increasing reliability in its core activity of packet exchange between its members. New projects are also constantly developed in order to facilitate peering between its members, and to research the peering ecosystem. NaMeX aims to foster the development of Internet networks and infrastructure in Southern Italy and the whole Mediterranean area. NaMeX joined Euro-IX, the European Internet Exchange Association, in 2003 [8].

${ }^{13}$ Founded in 2002, the Turin Piedmont Internet Exchange, Topix is an Internet Exchange characterized by a peculiar structure. Indeed, Topix is not just a single node, but an architecture stretching in the Piedmont and Valle d'Aosta regions with several interconnected access points (six in 2007). In this way Topix offers different possibilities of access, being also more fast and reliable. The second remarkable feature of Topix is its strategy. Topix is active in promoting the development of new technologies, and in particular has a remarkable role in favouring the broadband penetration [9].
}

peer at an IXP, an ISP usually has to establish a connection and pay a membership fee, then it can use the circuit to exchange traffic with all other members of the IXP willing to peer with it.

Earlier work [10] has identified several factors and problems that may affect networks' decision whether and with whom to peer. A first, rather obvious factor is size. Peering requires establishing bilateral traffic exchange points, or peering points, which entail fixed and variable technological costs. It follows that a sufficiently intense traffic flow between the end users of the two networks is a necessary precondition for peering to be economically viable. Since the costs of setting up and maintaining peering points are usually shared equally by peering networks, unbalanced traffic implies an unbalanced distribution of gains from peering against a balanced distribution of costs.

\subsection{Proximity and Interconnection Agreements}

Should we expect the geographical location of different ISPs to influence their interconnection decision? Of course, if two ISPs are very far away, building a connection from scratch would be very costly; hence one would expect that very far ISPs would not peer. However, we consider a situation where there is an IXP where interconnection is cheaper, and that there is a number of ISPs, all of which are already connected to this IXP. Should we then expect the geographical location of these ISPs to matter in their choice of traffic exchange partners?

Since [11] agglomeration has been attributed to three forces: a pooled labour market, greater provision of non-traded inputs, and knowledge spillovers. Reference [12] stressed the importance of geographic proximity in defining the extent of knowledge spillovers within firms of a given industry to explain the agglomeration in cities. Following this approach proximity matters since a basic input for firms' activities, tacit knowledge, is assumed to be only transferable through face to face interaction [13].

The role of proximity and face-to-face contact becomes more important as production processes become more fragmented and as firms have to rely on incomplete contracts, thus highlighting the importance of mutual trust $[14,15]$. This is particularly important for the Internet, where there is dominant asymmetric information involving the operators performances and commitment. In the interconnection decision between ISPs, and particularly for peering, there are substantial aspects of the transaction that are impossible to measure or monitor, so that the interconnection decision may require substantial trust and informal cooperation between linked partners. This may activate the centripetal force: face-to-face meetings and social connections may facilitate the gov- 
ernance of interconnection agreements, and the former may benefit from geographical proximity. Transport costs do not matter here since we consider a population of ISPs that are already connected to a given IXP and their only decision is whether or not to switch on an interface at an IXP were they are already located.

The specific environment where these issues are studied has however a clear characteristic: the Italian market for Internet broadband access where a large dominant operator provides connectivity to a large set of smaller ones. In this specific setting, proximity among the small may emerge as a crucial competitive advantage in interconnection.

The issues of agglomeration in the Internet, with a specific focus of the effect of proximity and asymmetry in interconnection decisions, have been addressed in [16] that introduced new metrics for asymmetry derived from Border Gateway Protocol (BGP) data and applied to the main IXP in United Kingdom. In this article we provide instead an analysis of the Italian situation, using both BGP and location data involving the three major Italian IXPs: Mix, Topix and Namex. As a result, we aim to characterize the agglomeration/polarization in the specific industry under study, defining the shape and borders, if existing, of its geographical distribution ${ }^{14}$.

In the following we present, for each of these IXPs, the map showing the distribution of interconnection agreements signed by its members. These maps, which are a tool to visualize the extent of agglomeration in the Italian Internet structure, are complemented by the estimation of spatial autocorrelation indexes. In the second part of this section we undertake a more formal analysis aimed to assess the actual role of geographical proximity for the interconnection decisions.

\subsection{Agglomeration in Interconnection}

The following Figure 2 depicts the distribution of interconnection agreements signed by the members of the Mix. Each column represents an ISP, where its height is proportional to the number of interconnection agreements signed by that ISP.

This map shows that almost all the Mix members are headquartered in Europe. More interestingly, by looking at the distribution of the bars, we see that there is no clear evidence of agglomeration in peering. This visual evidence is strengthened by the index of spatial autocorrelation [18], indicating a random pattern for the distribution of interconnection agreements ${ }^{15}$.

Very similar features characterise also the distribution of interconnection agreements within both Namex and Topix, characterised by a smaller number of members, as shown in Figures 3 and $\mathbf{4}$ below.

The Moran's I index of autocorrelation, as reported in Table 1 below, supports a random distribution for interconnection agreements at each of the three Italian IXPs:

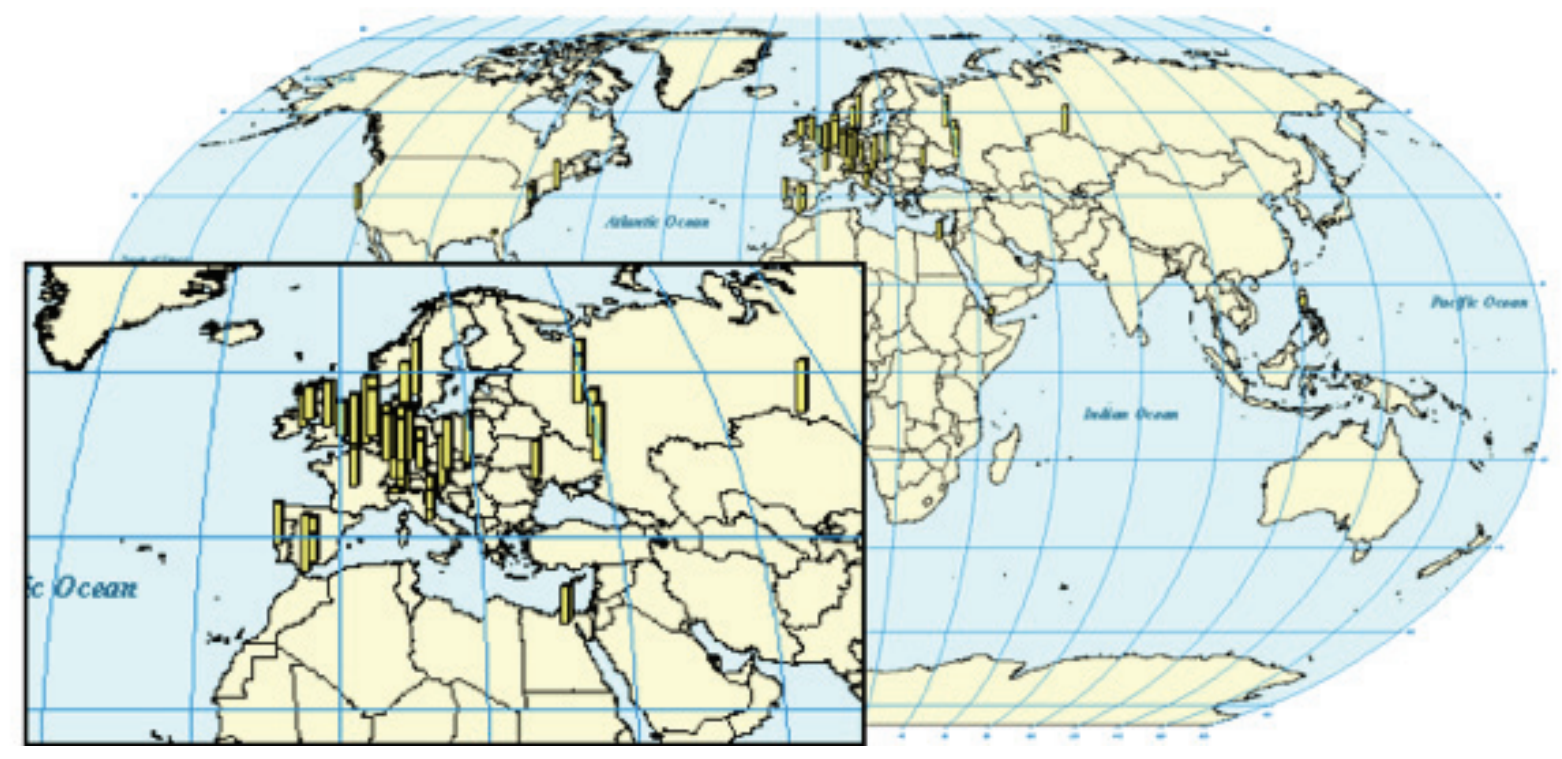

Figure 2. Interconnection geographical distribution at the MIX.

\footnotetext{
${ }^{14}$ Reference [17] addressed agglomeration when ICT becomes a substitute of face-to-face dealings. In an analysis of the Emilia Romagna's industrial districts, he found that spatial concentration is no longer the crucial factor in agglomeration and introduces the term 'multi-located' district to describe agglomeration.

${ }^{15}$ The Moran's I index is used to test the hypothesis of no-clustering for spatially distributed variables. This index, measuring spatial autocorrelation, is calculated by considering the value of the variable under analysis at different locations, see [19]. The $\mathrm{z}$ statistic is employed to test the hypothesis of no spatial correlation, and the sign of the geographical clustering: a positive value for the $\mathrm{z}$ statistic suggests positive spatial autocorrelation - clustered outcome - while a negative value for the $\mathrm{z}$ statistic a dispersed one.
} 


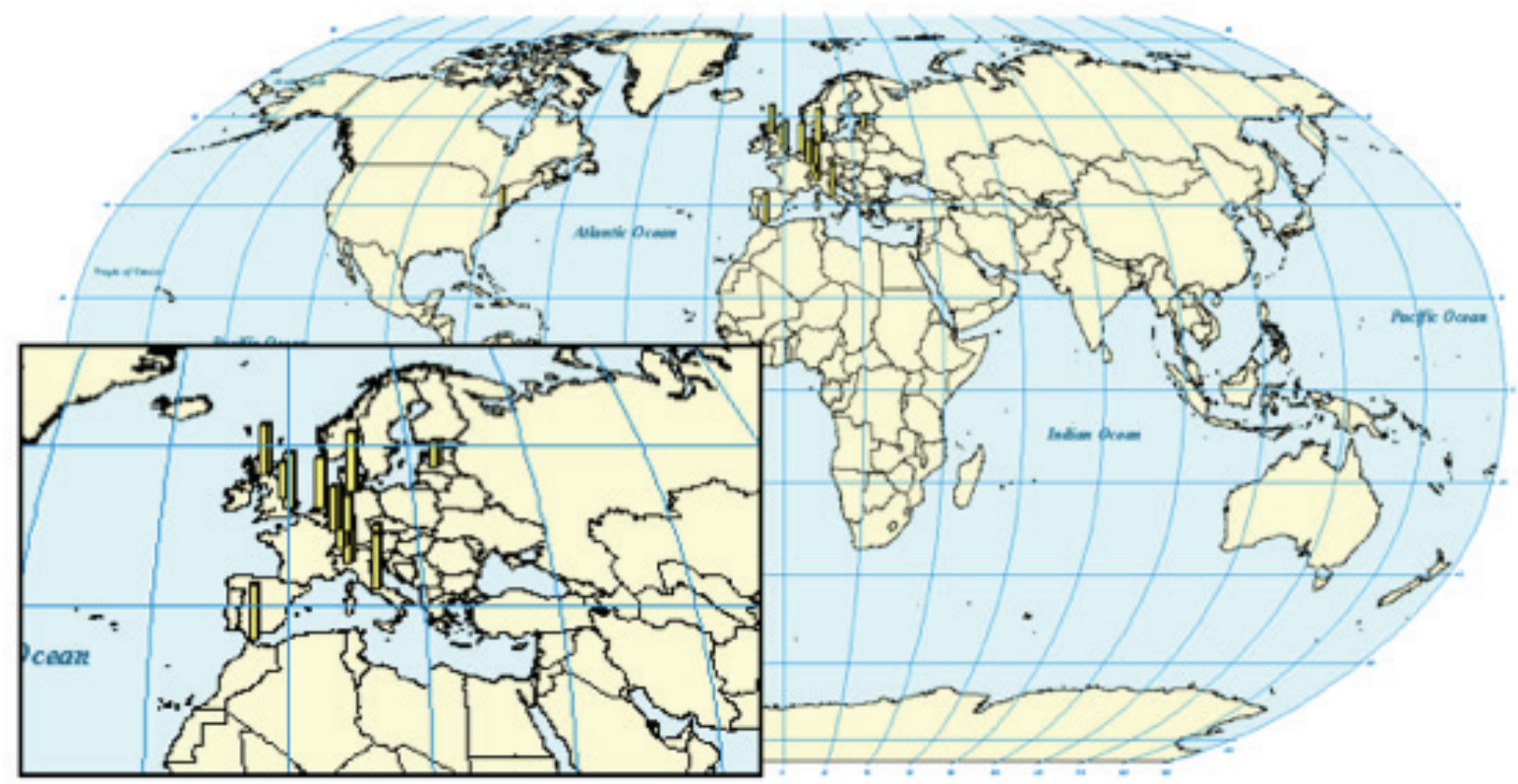

Figure 3. Interconnection geographical distribution at the NAMEX.



Figure 4. Interconnection geographical distribution at the TOPIX.

Table 1. Spatial autocorrelation statistics for interconnection agreements distributions for the main Italian IXPs.

\begin{tabular}{ccccc}
\hline $\begin{array}{c}\text { IXP } \\
\text { (Location) }\end{array}$ & $\begin{array}{c}\text { Moran's I } \\
\text { index }\end{array}$ & $\begin{array}{c}\text { Theoretical I under the } \\
\text { hypothesis of no spatial } \\
\text { autocorrelation }\end{array}$ & z-value & Outcome \\
\hline $\begin{array}{c}\text { MIX } \\
\text { (Milan) }\end{array}$ & 0.014 & -0.018 & 1.067 & random \\
$\begin{array}{c}\text { NAMEX } \\
\text { (Rome) }\end{array}$ & -0.153 & -0.077 & -0.839 & random \\
$\begin{array}{c}\text { TOPIX } \\
\text { (Turin) }\end{array}$ & -0.077 & -0.091 & 0.147 & random \\
\hline
\end{tabular}

Mix, Namex and Topix.

From an IXP based point of view there is no agglomeration in interconnection. It is interesting to notice, however, that the distribution of the relevant IXPs in Italy is still unbalanced across its territory.

\subsection{Proximity and Interconnection}

We now move to the analysis of the effect of geographical proximity on the interconnection decisions. We estimate a binary (probit) model. The dependent variable, 
the interconnection decision, is obtained from the peering matrix for each IXP: this is a symmetric matrix showing, for each pair of providers members of the IXP, whether these are directly interconnected or not.

The explanatory variables are introduced to model: the extent of geographical proximity ${ }^{16}$, the strength of mutual knowledge and reputation effects, the magnitude of imbalances in both size and traffic flows as well as technical elements.

A second set of variables was obtained from the distribution of the providers' memberships across the IXPs of the Euro-IX ${ }^{17}$ association.

The possibility of reputation effects in interconnection decisions [21] is expressed by a variable indicating, for each pair of providers, how many IXPs of the EURO-IX they are both members of. Secondly, we introduce a variable to model the asymmetry in the providers' size by considering the difference in the number of Euro-IX IXPs memberships every ISP has. Finally, we devised a variable to model traffic imbalances. Although traffic flows are kept confidential, it is possible to determine a proxy for traffic imbalances by looking at the publicly available Border Gateway Protocol (BGP) routing tables.

In particular, we calculated the following measure of betweenness centrality [22] for each Internet operator $v$ :

$$
B_{s}(v)=\sum_{s \neq v \neq t \in V} \sigma_{s t}(v)
$$

where: $\sigma_{s t}(v)=\sigma_{t s}(v)$

is the number of shortest BGP paths from the Internet operator $\mathrm{s}$ to the operator $\mathrm{t}$ on which the $v$ lies on. High betweenness for $v$ indicates that presumably a relevant

${ }^{16}$ The geographical distance between any pair of providers was calculated following a two-stage strategy: firstly every provider was identified through its latitude and longitude coordinates, then these data were used in the great circle distance formula to obtain the distance.

${ }^{17}$ Euro-IX (European Internet Exchange Association) was formed in May 2001 with the intention to further develop, strengthen and improve the IXP community. A number of IXPs recognised a need to combine their resources so as to co-ordinate technical standards across the continent, develop common procedures, and share and publish statistics and other information. This publishing of information would in turn give all interested parties a better insight into the world of IXPs. The IXPs members are: AIX Athens Greece; AMS-IX Amsterdam Netherlands; BCIX Berlin Germany; BIX Budapest Hungary; BNIX Brussels Belgium; CATNIX Barcelona Spain; CIXP Geneva Switzerland; DE-CIX Frankfurt am Main Germany; Equinix 11 international locations United States; ESPANIX Madrid Spain; FICIX Helsinki Finland; GIGAPIX Lisbon Portugal; GN-IX Groningen Netherlands; INEX Dublin Ireland; JPIX Tokyo, Nagoya, Osaka Japan; JPNAP Tokyo, Osaka Japan; LINX London United Kingdom; LIPEX London United Kingdom; LIX Luxembourg Luxembourg; LONAP London United Kingdom; MaNAP Manchester United Kingdom; MIX Milan Italy; MSK-IX Moscow Russia; NaMeX Rome Italy; NDIX Enschede, Munster (DE), Dusseldorf (DE) Netherlands; Netnod Stockholm Sweden; NIX Oslo Norway; NIX.CZ Prague Czech Republic; NIXI Delhi, Mumbai, Chenna, Kolkata India; NOTA Miami United States; PARIX Paris France; RoNIX Bucharest Romania; SIX Ljubljana Slovenia; Switch and Data 23 locations United States; TIX Zurich Switzerland; TIX Tuscany Florence Italy; TOPIX Torino Italy; VIX Vienna Austria, [20]. quantity of traffic flows among that node. This variable is arguably the most reliable proxy in quantifying network asymmetries.

In the following we introduce the probit model estimation results for Mix, Namex and Topix.

The above table shows that two variables seem to consistently affect the interconnection decision between a pair of providers. These are the geographical distance and the ISPs size's asymmetry, captured through the betweenness. Geographical proximity positively affects the likelihood of interconnection; this result is interesting since it shows that even within the Internet sector, usually considered to be driving the death of distance process,

Table 2. Probit regression model variables.

\begin{tabular}{|c|c|}
\hline \multicolumn{2}{|r|}{ dependent variable } \\
\hline $\begin{array}{l}\text { Interconnection } \\
\quad \text { (dummy) }\end{array}$ & $\begin{array}{l}\text { Assumes value } 1 \text { in case of interconnection } \\
\text { between providers, } 0 \text { otherwise. }\end{array}$ \\
\hline \multicolumn{2}{|r|}{ independent variables } \\
\hline Dist ISPs & $\begin{array}{c}\text { distance between each couple of ISPs (in } \\
\text { thousands of miles) }\end{array}$ \\
\hline Common_IXPs & $\begin{array}{l}\text { number of European IXPs in which the } \\
\text { ISPs are both present }\end{array}$ \\
\hline Diff_IXP_Member & $\begin{array}{c}\text { difference in the number of memberships in } \\
\text { European IXPs }\end{array}$ \\
\hline Diff_Relevance & $\begin{array}{l}\text { difference in the betweenness value (in } \\
\text { hundreds thousands of units) }\end{array}$ \\
\hline
\end{tabular}

Table 3. Probit estimated model results.

\begin{tabular}{lccc}
\hline & Mix & Namex & Topix \\
\hline Dist ISPs & -0.047 & -0.164 & -0.62 \\
& $(20.17)$ & $(1.40)$ & $(2.82)$ \\
Common_Ixp & 0.245 & 0.335 & - \\
& $(5.65)$ & $(0.93)$ & - \\
Diff_IXP_Member & -0.059 & 0.236 & 0.710 \\
& $(4.99)$ & $(0.97)$ & $(2.11)$ \\
Diff_Relevance & -0.003 & -0.007 & -0.108 \\
& $(7.22)$ & $(1.54)$ & $(1.44)$ \\
Constant & 0.335 & 0.342 & 0.362 \\
& $(4.62)$ & $(0.56)$ & $(1.15)$ \\
Observations & 1275 & 91 & 56 \\
Pseudo R-Square & 0.1053 & 0.0575 & 0.2417 \\
Log-likelihood & -774.4 & -49.4 & -29.3 \\
Significance test statistic: LR chi2 & $\begin{array}{l}182.21 \\
\text { chi2(4) }\end{array}$ & $\begin{array}{l}6.04 \\
\text { chi2(4) }\end{array}$ & $\begin{array}{l}18.70 \\
\text { chi2(3) }\end{array}$ \\
Absolute value of z statistics in parentheses & & \\
\hline
\end{tabular}


there is still a role for proximity effects and therefore for the emergence of a non-virtual type of district morphology in the most of virtual economic activities: the routing of Internet traffic along non dedicated inter-providers connectivity paths. Indeed, interconnection agreements also rely on mutual trust: for instance, peering agreements (but not transit) do not have Service Level Agreements (SLAs) that guarantee rapid repair is a problem occurs. As argued earlier mutual knowledge seems to play a role too. The variable Common IXPs, indicating for each pair of providers the number of other IXPs where are both present, positively affects interconnection, although the variable is not statistically significant for Namex.

The second consistent result is the negative, and expected, effect of network asymmetries on interconnection.

\section{Conclusions}

In this paper we investigated the extent of agglomeration characterizing the Internet Industry in Italy by analyzing the interconnection decisions between Internet Service Providers participating at the three main Internet Exchange Points (Mix, Topix and Namex).

Firstly we focused on a static analysis, providing the picture of the emerging morphology in interconnection. In particular we provided a series of maps depicting the distribution of interconnection agreements, complemented by the correspondent spatial autocorrelation statistics. Results showed a dominant presence of random patterns, suggesting a non-agglomerated equilibrium. This result is interesting given the clear heterogeneity in the Internet penetration in Italy. However, we also notice that the distribution of IXPs is still unbalanced in the territory.

We then investigated the possibility that distance still plays a role in the strategic interconnection decisions between providers. Indeed, the possibility of mutual control is argued to be important for the governance of relationships, such interconnection, characterized by highly asymmetric information.

Interestingly, geographical distance is significant in explaining interconnection, having a negative effect on its likelihood: this may indicate relevance for mutual knowledge and face-to-face contact in interconnection decisions. Our interpretation of this role played by proximity relates it to the specifically concentrated market situation in Italy where a dominant operator still manages large shares of the final user's broadband traffic. Smaller operators, often need to join efforts to contrast the dominance of the largest ones that, as shown by the effect of size asymmetry, are also in general unwilling to peer with the small ones. This necessity of cooperation among smaller competitors jointly facing a dominant former monopolist shows also at an institutional level as for example with the role played by the Italian Association of Service Providers and through the increasing relevance of cooperative, non-profit organizations as the IXPs. The need for reciprocal direct interconnection in the still concentrated Italian broadband access market, might explain the relevance of geographical proximity in maintaining cooperation among small competing providers of Internet access in Italy.

\section{Acknowledgements}

The authors would like to thank Valeria Rossi, from MIX for numerous and inspiring discussions, the editor of Modern Economy and two anonymous referees for their suggestions, and the participants to the 18th European Regional ITS Conference and of the 35th Conference of the European Association for Research in Industrial Economics, where earlier versions of the paper were discussed. The final responsibility for any mistake remains however only ours.

\section{References}

[1] European Commission "Commission Staff Working Document, Accompanying the Communication from the Commission to the European Parliament, the Council, the European Economic and Social Committee and the Committee of the Regions, Progress Report on the Single European Electronic Communications Market," Council of the European Union, Brussels, 22 May 2009.

[2] J. G. Sidak "A Consumer-Welfare Approach to Network Neutrality Regulation of the Internet," Journal of Competition Law and Economics, Vol. 2, No. 3, 2006, pp. 349-474. doi:10.1093/joclec/nh1016

[3] S. Laitner, "Telecoms Groups Attack Shake-Up Plan," Financial Times, Brussels, 14 November 2007.

[4] European Commission "Commission Staff Working Document Annex to the Communication from the Commission to the European Parliament, the Council, the European Economic and Social Committee and the Committee of the Regions, European Electronic Communications Regulation and Markets" Commission of the European Communities, Brussels, 29. March 2007.

[5] BT Group plc Annual Report \& Form 20-F, 2008. http://www.btplc.com/Report/Report08/pdf/AnnualRepor t2008.pdf

[6] M. Merola and S. Lembo, "Antitrust Encyclopedia: Italy," February 2009. www.concurrences.com/nr_pays.php3?liste_pays $=15$

[7] The Milan Internet Exchange website, 2007. www.mix-it.net

[8] The Nautilus Mediterranean Exchange Point Website, 
2007.

www.namex.it/

[9] The Torino Piemonte Internet Exchange Point Website, 2007. http://www.top-ix.org/.

[10] A. D'Ignazio and E. Giovannetti, "From Exogenous to Endogenous Networks: Internet Applications," Journal of Economic Surveys, Vol. 20, No. 5, 2006, pp. 757-796. doi:10.1111/j.1467-6419.2006.00267.x

[11] A. Marshall, "Principles of Economics," Macmillan, London, 1890.

[12] E. Glaeser, H. Kallal, J. Scheinkman, and A. Schleifer, "Growth of Cities," Journal of Political Economy, Vol. 100, No. 6, 1992, pp. 1126-1152. doi:10.1086/261856

[13] M. Fujita and J. F. Thisse, "Economics of Agglomeration: Cities, Industrial Location, and Regional Growth," Cambridge University Press, Cambridge, 2002.

[14] G. Spagnolo, "Social Relations and Cooperation in Organizations," Journal of Economic Behavior and Organization, Vol. 38, No. 1, 1999, pp. 1-26. doi:10.1016/S0167-2681(98)00119-X

[15] E. Learner and M. Storper, "The Economic Geography of the Internet Age," The National Geography of the Economic Research, NBER Working Paper, No. 8450, Cam- bridge, 2001.

[16] A. D'Ignazio and E. Giovannetti, “Asymmetry and Discrimination in Internet Peering, Evidence from the LINX," International Journal of Industrial Organization, Vol. 27, 2009, pp. 441-448. doi:10.1016/j.ijindorg.2008.11.004

[17] E. Santarelli, "Patents and the technological Performance of District Firms Evidence for the Emilia-Romagna Region of Italy," Max Planck Institute for Research into Economic Systems Papers on Entrepreneurship, Growth and Public Policy, No. 29, 2004.

[18] P. Moran, "The Interpretation of Statistical Maps," Journal of the Royal Statistical Society, Vol. 10, 1948, pp. 243-251.

[19] G. J. G., Upton and B. Fingleton, "Spatial Data Analysis by Example. Volume 1: Point Pattern and Quantitative Data," John Wiley \& Sons Ltd., Chichester, 1985.

[20] The European Internet Exchange Association website. http://www.euro-ix.net/, 2007.

[21] N. Titley "An Analytical Model of Peering between Internet Service Providers," Release 1, unpublished, 1997.

[22] A. Shimbel, "Structural Parameters of Communication Networks," Bulletin of Mathematical Biophysics, Vol. 15, 1953, pp. 501-507. doi:10.1007/BF02476438 